\title{
Reflujo gastroesofágico en pediatría
}

\author{
GLADYS GUEVARA P. ${ }^{1}$, MARCELA TOLEDO C. ${ }^{2}$, RAMA DE GASTROENTEROLOGÍA \\ DE LA SOCIEDAD CHILENA DE PEDIATRÍA \\ 1. Profesor Asistente Departamento de Pediatría y Cirugía Infantil Campus Centro Universidad de Chile, \\ Gastroenterólogo Infantil. Unidad de Gastroenterología Infantil Hospital Clínico San Borja-Arriarán. \\ 2. Gastroenterólogo Infantil, Hospital Roberto del Río.
}

\begin{abstract}
Gastro esophageal reflux disease in pediatrics

Gastro Esophageal Reflux (GER) is the passage of gastric content to the esophagus with or without vomitus or regurgitation. GER is a physiological process that occurs in $50 \%$ of newborns, spontaneously resolving at 12 to 14 months of age. When this retrograde gastric content reaches the esophagus and produces uncomfortable symptoms or complications, a disease is produced, named Gastro Esophageal Reflux Disease or GERD. Reflux episodes occur due to transient relaxation of the inferior esophagic sphincter, triggered by distension of the gastric fundus. Nursing children show higher frequency of episodes of relaxation due to a higher frequency of feeding than older children do. Most frequent symptoms include frequent regurgitation, with out without vomit, weight loss or poor weight gain, irritability, pyrosis, hematemesis, dysphagia, odinophagia, sibillances, stridor, cough, dysphonia, among others. These are very inespecific symptoms. Studies for GERD include 24 $\mathrm{H}$ pHmeasurement, which permits the study of associatrion between one of these symptoms and reflux, as well as the study of those patients with poor response to medical treatment. Unfortunately, it does not detect non-acid reflux. Multichannel intraluminal $\mathrm{pH}$ impedance measurement is a relatively new exam that measures the movement of fluids, gas or solids by a change in electric impedance through the esophagus. It allows to detect small volumes, type of content, acid and non acid and correlated with symptoms of GERD. Other useful exams include endoscopy with biopsy of the esophagus for diagnosis of esophagitis, esophageal mannometry for specific cases and gastroesophagic scintigram for pulmonary aspiration. Treatment include change in lifestyle (feeding, position, habits), pharmacological treatment and surgery. Medical treatment includes antacids, PPIs, most frequently omeprazole, $\mathrm{H} 2$ blockers such as ranitidine have a lower rate of healing of esophagitis than omeprazole, and appears to show tachyphylaxis after 6 weeks of treatment. Antireflux surgery has very specific indications, shows morbidity and mortality, and recurrence of symptoms.

(Key words: Physiological gastroesophageal reflux, gastroesophageal reflux disease, lower esophageal sphincter).

Rev Chil Pediatr 2011; 82 (2): 142-149
\end{abstract}

Trabajo recibido el 01 de abril de 2011, aceptado para publicación el 29 de abril de 2011.

Correspondencia a:

Gladys Guevara P.

E-mail: cguevara@mi.cl 


\section{RESUMEN}

El reflujo gastroesofagico (RGE) es el paso del contenido gástrico hacia el esófago con o sin vómitos o regurgitación. El RGE es un proceso normal fisiológico que ocurre en el 50\% de los lactantes, resolviéndose espontáneamente a los 12 a 14 meses de edad. Cuando este contenido retrógrado gástrico alcanza al esófago y produce síntomas molestos o complicaciones se produce la enfermedad por RGE o ERGE. Los episodios de reflujo se producen principalmente por relajaciones transitorias del esfínter esofágico inferior (LES) gatillado por distensión del fondo gástrico. Los lactantes presentan mayor numero de episodios de relajación del LES debido a que se alimentan con más frecuencia que los niños mayores. Los síntomas más frecuentes asociados con reflujo patológico son regurgitaciones frecuentes con o sin vómitos, pérdida de peso o pobre ganancia de peso, irritabilidad, pirosis, hematemesis, disfagia, odinofagia, sibilancias, estridor, tos, disfonía entre otros, estos síntomas son poco específicos en lactantes y niños menores. Los exámenes para el estudio de ERGE son la pH metría de 24 hrs que permite estudiar la asociación entre un síntoma y el reflujo, también es útil para estudiar aquellos pacientes con mala respuesta al tratamiento médico, sin embargo, tiene el inconveniente que no detecta episodios de reflujo no ácidos. La pH-Impedanciometria Intraluminal multicanal (pH IIM) es un examen relativamente nuevo mide los movimientos de fluidos, gas o sólidos a través de cambios en la impedancia eléctrica a lo largo del esófago; permite distinguir pequeños volúmenes refluidos y el tipo de contenido, detecta reflujo acido y no acido y correlaciona síntomas con ERGE. Otros exámenes útiles son la endoscopia con biopsia de esófago para estudio de esofagitis. La Manometría esofágica tiene sus indicaciones específicas y la Cintigrafía gastroesofágica que es otro examen que se utiliza principalmente para estudiar aspiración pulmonar. El tratamiento incluye cambios en el estilo de vida, terapia farmacológica y la cirugía. Dentro de los cambios del estilo de vida se deben considerar cambios en la alimentación, posición y de hábitos en los niños mayores. En el tratamiento médico se utilizan principalmente los medicamentos antiácidos como los inhibidores de la bomba de protones dentro de los cuales el omeprazole es el más utilizado. De los medicamentos antagonistas de los receptores histamina-2, la ranitidina tiene una menor tasa de curación de la esofagitis en comparación con el omeprazol, se ha descrito taquifilaxia posterior a las seis semanas de tratamiento. La cirugía anti reflujo tiene indicaciones específicas, se han descrito recurrencia de síntomas y no está exenta de complicaciones y morbilidad.

(Palabras clave: Reflujo gastroesofagico fisiológico, enfermedad por reflujo, esfínter esofágico inferior). Rev Chil Pediatr 2011; 82 (2): 142-149

El reflujo gastroesofagico (RGE) es el paso del contenido gástrico hacia el esófago con o sin vómitos o regurgitación, esta última se diferencia del vómito porque se realiza sin esfuerzo y no se presenta en forma explosiva, aunque a veces en el lactante menor puede ser muy intensa. El RGE es un fenómeno fisiológico que ocurre en todas las edades pero principalmente en el lactante, en que la mayoría de los episodios de RGE duran menos de tres minutos, ocurren en período post prandial y cursan con pocos o ningún síntoma. La regurgitación se produce a diario en el 50\% de los niños menores de tres meses y es el síntoma más visible del RGE madurativo o fisiológico, que generalmente se resuelve espontáneamente en la mayoría de los lactantes sanos a los 12 a 14 meses de edad.

Cuando este contenido retrógrado gástrico alcanza al esófago y produce síntomas molestos o complicaciones se produce la enfermedad por RGE o ERGE. El material refluido puede ser aire, líquido sólido o una mezcla de todos y dependiendo del $\mathrm{PH}$ puede ser ácido $(\mathrm{PH}<4)$ o no ácido. Se sabe que los episodios de reflujo no ácido son tan frecuentes como los episodios ácidos, pudiendo estos también ser sintomáticos.

El esfínter esofágico inferior (LES) es la barrera anti reflujo más importante, el LES es una zona de alta presión mantenida por dos sistemas musculares: el primero está constituido por una capa de musculo liso y el segundo por una capa de musculo estriado. El LES se ubica normalmente en la cavidad abdominal, bajo el diafragma y la presión abdominal que es mayor que la presión del tórax permite el mecanismo de esfínter del LES. El desplazamiento 
del LES a la cavidad torácica que se produce en la hernia hiatal altera el funcionamiento normal del LES y favorece la ERGE.

Una vez que el material refluido entra en el esófago, el bolo alimentario causa distención de la pared, y estimula receptores los cuales inducen una contracción mediada neurológicamente que mueve el material refluido hacia el estómago. Esta perístalsis secundaria es suplementada por una onda peristáltica primaria inducida por la deglución que permite limpiar el material refluido; esta onda de limpieza o clearance es importante para prevenir el daño de la mucosa esofágica por el contacto prolongado con el material refluido. Otra barrera fisiológica para evitar el daño de la mucosa esofágica por el reflujo es el reflejo de deglución de saliva alcalina en respuesta al episodio de RGE que permite neutralizar el contenido ácido del material refluido, estos dos últimos reflejos no están presentes durante el sueño, por lo tanto, los episodios de reflujo nocturnos son más dañinos para la mucosa esofágica.

Los episodios de reflujo ocurren con mayor frecuencia durante períodos transitorios de relajación del esfínter esofágico inferior (LES), esta breve relajación del LES puede ser gatillada por distensión del fondo gástrico y es mediada por el nervio vago. La relajación transitoria del LES puede resultar en reflujo de aire, sólido o gas o una mezcla de contenido gástrico. En lactantes los episodios de reflujo son más frecuentes y debidos a alimentaciones frecuentes que distienden el fondo gástrico causando más eventos de relajación del LES. Una menor proporción de episodios de reflujo se produce cuando la presión del LES no aumenta, cuando se produce un aumento repentino de la presión intraabdominal o cuando la presión del LES en reposo esta disminuida en forma crónica. Las alteraciones en varios de los mecanismos de protección tales como: clearance insuficiente y amortiguación de contenido refluido, retraso del vaciamiento gástrico, anormalidades en la restitución del epitelio, reparación y disminución de los reflejos neuronales de protección del tracto aereo digestivo permiten que el RGE fisiológico se transforme en enfermedad por reflujo gastroesofágico (ERGE).

Varios síntomas de reflujo patológico, her- nia hiatal, esofagitis, esófago de Barret y adenocarcinoma, ocurren en la misma familia lo que sugiere la presencia de un componente hereditario en la enfermedad por reflujo y sus complicaciones.

Existen grupos de pacientes pediátricos que tienen mayor incidencia de reflujo patológico como: pacientes con enfermedades neurológicas, obesos, ciertos síndromes genéticos, atresia esofágica, enfermedades pulmonares crónicas, y aquellos pacientes con antecedentes de ser recién nacido prematuro.

La mayoría de los síntomas de reflujo son poco confiables en los menores de 8 a 12 años de edad y son poco específicos en lactantes y niños menores. Generalmente la sintomatología de ERGE varía con la edad y tiene relación con patologías subyacentes.

Los síntomas más frecuentes asociados con reflujo patológico son regurgitaciones frecuentes con o sin vómitos, pérdida de peso o pobre ganancia de peso, irritabilidad, pirosis, hematemesis, disfagia, odinofagia, sibilancias, estridor, tos, disfonía entre otros. La presencia de: estenosis esofágica, esófago de Barret, laringitis, neumonía a repetición, anemia crónica, erosiones dentales, síndrome de Sandifer, episodios de apnea o ALTE debe hacer pensar en un RGE patológico como una de las posibles causas.

Se ha visto una relación probable entre RGE y apnea obstructiva en aquellos casos en quienes el episodio ocurre cuando el paciente está durmiendo, en posición supina y dentro de una hora post alimentación, aunque no se ha demostrado de manera fehaciente una relación temporal entre acidificación del esófago y apnea.

La importancia del enfoque en la anamnesis y examen físico es detectar otras patologías que se presentan con vómitos y regurgitación e identificar complicaciones del ERGE. Los signos de alarma en el paciente vomitador son:

- Inicio de los vómitos posterior a los 6 meses de edad.

- Vómitos biliosos.

- Hematemesis, hematoqueccia.

- Mal incremento de peso.

- Diarrea, constipación. 
- Fiebre, letargia.

- Hepatoesplenomegalia.

- Fontanela abombada, macro y microcefalia, convulsiones.

- Dolor abdominal y/o distención abdominal etc.

\section{Exámenes para estudio de RGE}

\section{pHmetría}

La monitorización del pH esofágico intraluminal se realiza a través de 1 catéter, de antimonio o fibra de vidrio, de inserción nasal con 1 o más electrodos colocados a lo largo, con el objetivo de capturar, y analizar los eventos de reflujo ácido. Se considera un episodio de reflujo ácido cuando el $\mathrm{pH}$ es inferior a 4.0

Los parámetros obtenidos de la $\mathrm{pH}$ metría incluyen:

- Número total de episodios de reflujo.

- Número de reflujos mayor a 5 minutos de duración.

- Duración del episodio de reflujo más largo.

- El índice de reflujo (IR): porcentaje del total del registro en que el $\mathrm{pH}$ es menor a 4. Es considerado anormal un IR > a 7 y normal cuando es menor a 3 entre 3 y 7 indeterminado.

Se puede evaluar además si los episodios de reflujo ocurren en posición de pie, acostado, durmiendo o despierto, y su relación con la alimentación.

El índice de reflujo es el parámetro más importante y que resume el resultado del registro. Sin embargo, no existe correlación entre la severidad del reflujo y los hallazgos de la pH metría, tampoco permite predecir con ésta las posibles complicaciones de la enfermedad por reflujo.

La pH metría es útil para evaluar la respuesta a la terapia anti secretora. Permite determinar la asociación temporal entre el reflujo ácido y frecuencia de un síntoma como tos y en los asmáticos o sibilantes detectar reflujo ácido que puede ser un factor agravante de sus crisis. Sin embargo, adolece de captar los episodios de reflujos no ácidos postprandiales.

\section{Impedanciometría intraluminal múltiple MII y pH metría}

Es un procedimiento que mide los movimientos de fluidos, sólidos y aire en el esófago. Mide cambios en la impedancia eléctrica (resistencia) entre los diferentes electrodos colocados a lo largo del catéter esofágico.

$\mathrm{Al}$ existir un evento de reflujo hay un cambio de impedancia en el canal distal del contenido refluido hacia el canal proximal. Se puede calcular la dirección y la velocidad del bolo, por la distancia definida entre los canales y el tiempo entre las alteraciones de los patrones de impedancia de los pares secuenciales de electrodos.

Distingue pequeños volúmenes refluidos y el tipo de contenido.

La combinación de Impedanciometría y pH metría en un mismo catéter da información adicional, por ejemplo, si el material refluido es ácido o no, sobre todo en los períodos postprandiales en que el contenido gástrico es no ácido. La asociación de este examen con la monitorización de síntomas, o video polisomnograma permite la correlación de síntomas como apnea, tos y otros síntomas respiratorios. Tiene el inconveniente que los valores normales para las diferentes edades no han sido establecidos.

La impedanciometría con pHmetría permite detectar: reflujo ácido y no ácido, altura del material que refluye (RGE y síntomas respiratorios), contenido y dirección del material que refluye (líquido, aire o mixto).

Es un buen examen para evaluar severidad, pronóstico y respuesta al tratamiento.

\section{Manometría esofágica}

Mide peristaltismo esofágico, presión de LES y Esfínter esofágico superior (EES) y la coordinación de estructuras durante la deglución.

Ha tenido un rol importante en identificar a las relajaciones transitorias del LES como causa de Enfermedad por reflujo (ERGE).

Está indicada en: falla en terapia supresora de ácido, EDA negativa con posible trastorno de motilidad esofágica, determinar posición de LES (pH), confirmar diagnóstico de acalasia y otros desórdenes motores. 


\section{Biopsia y endoscopia}

La endoscopia permite la visualización directa de la mucosa esofágica y la biopsia la anatomía microscópica de su mucosa

Las lesiones encontradas en la enfermedad por reflujo son: erosiones, con o sin fibrina, úlceras, estenosis, áreas de posible metaplasia de mucosa gástrica o intestinal (Barret) y pólipos. También permite hacer el diagnóstico de anomalías anatómicas que predispongan a RGE patológico como es la hernia hiatal.

La definición de esofagitis a través de la endoscopia debe consignar la presencia de lesiones en la mucosa esofágica o inmediatamente sobre él LES. Existen clasificaciones de lesiones esofágicas para niños (clasificación de los Angeles) que son útiles de usar para unificar hallazgos inter observador. Sin embargo si no hay lesiones no se puede descartar la presencia de enfermedad por reflujo no erosiva.

\section{Clasificación de la esofagitis de Los Angeles}

- GRADO A: una o más lesiones mucosas, menores de $5 \mathrm{~mm}$, que no confluyen en los extremos superiores de dos pliegues mucosos.

- GRADO B: una o más lesiones mucosas, mayores de $5 \mathrm{~mm}$, que no confluyen en los extremos superiores de dos pliegues mucosos.

- GRADO C: una o más lesiones mucosas, confluyentes entre pliegues mucosos, pero que ocupan menos del $75 \%$ de la circunferencia esofágica.

- GRADO D: una o más lesiones mucosas, confluyentes entre pliegues mucosos, que ocupan al menos el $75 \%$ de la circunferencia esofágica.

Las muestras deben ser de buen tamaño, orientación y a diferentes niveles ya que las lesiones histológicas en la Enfermedad por RGE son en parche. Se puede describir eosinofilia, elongación de las papilas, hiperplasia basal mayor del 20 al 25\% del total del epitelio, espongiosis (dilatación intercelular), hallazgos que no son específicos, pueden estar influen- ciados por medicamentos usados para el tratamiento de la esofagitis u otras enfermedades.

El rol más importante de la histología es realizar el diagnóstico diferencial de otras enfermedades que simulan ERGE como esofagitis eosinofílica, Esófago de Barret, Enfermedad de Crohn o infecciones esofágicas.

\section{Radiografía esófago estómago duodeno}

La Radiografía EED (Rx EED) contrastada con bario, tiene un rol en detectar alteraciones anatómicas como fistula, mal rotación, estenosis pilórica, estenosis, acalasia, consideradas diagnóstico diferencial de RGE. El examen tiene una sensibilidad de $31-86 \%$ y especificidad del 21-83\%. Tiene falsos positivos y falsos negativos, por la corta duración del examen. No se justifica realizar series radiológicas para el diagnóstico, ni para evaluar la gravedad del reflujo.

\section{Cintigrafía}

Consiste en marcar con tecnecio 99 alimentos o fórmula láctea la que es detectada en las áreas de interés como esófago, estómago, duodeno y pulmón. Esta técnica evalúa la presencia de reflujo post prandial independiente del PH gástrico y a diferencia de otros exámenes detecta reflujo no ácido.

Brinda información sobre el tiempo del vaciamiento gástrico, el que puede estar retardado en ERGE. No existe una estandarización en los valores normales por edad.

La cintigrafía gastroesofágica detecta episodios de reflujo y aspiración durante un período corto después de la alimentación. Los eepisodios de aspiración se pueden detectar a la hora y hasta las 24 hrs del inicio del examen, sin embargo, tiene baja sensibilidad para detectar las micro aspiraciones. Un test negativo, no excluye la posibilidad que existan aspiraciones. La sensibilidad de este test es baja (15 a 59\%) y la especificidad es alta (33 a 100\%).

Es un examen no indicado de rutina para el diagnóstico o manejo de RGE, pero si cobra importancia cuando se sospecha retención gástrica.

\section{Ultrasonografia}

Es una técnica no recomendada para el 
diagnóstico y manejo de RGE en niños. No brinda mayor información que lo obtenido por otras técnicas y no tiene correlación con los episodios de RGE detectados por pHmetría.

\section{Tratamiento}

El tratamiento incluye:

1. Cambios en el estilo de vida

2. Terapia farmacológica

3. Cirugía

\section{Cambios en el estilo de vida:}

Se refiere a los cambios en la alimentación, la posición y a los hábitos en los mayores.

En el Lactante:

- Alimentación

Tipo de fórmula: los lactantes alimentados con pecho o fórmula tienen similar número de episodios de reflujo fisiológico, sin embargo, con leche materna estos eventos serían más cortos.

Cuando se sospecha alergia a la proteína leche de vaca hay estudios que proponen el uso de leches altamente hidrolizadas por 15 días hasta 4 semanas, o bien la restricción de lácteos, soya y huevo a la madre. Sin embargo, no está claro la alergenicidad de las fórmulas clásicas y su relación con la manifestación de reflujo por lo que es posible que produzca riesgos en la alimentación a largo plazo. Se requieren más estudios.

Uso de espesantes: decrecen el número de regurgitaciones mejorando la calidad de vida del paciente, pero no se ha demostrado que sean útiles en la mejoría clínica de la ERGE.

Fraccionamiento: al fraccionar y disminuir los volúmenes de la fórmula se disminuyen los episodios de reflujo.

- Posición:

En los estudios con pHmetría la posición prona disminuye la cantidad de ácido del esófago en comparación con la posición supina. Sin embargo, el decúbito prono está asociado con una mayor incidencia de síndrome de muerte súbita del lactante (SMSL).

El riesgo de SMSL supera el beneficio de dormir boca abajo en el RGE, por lo tanto, se recomienda la posición supina durante el sueño en la mayoría de los bebés desde el nacimiento hasta los 12 meses de edad.

La posición decúbito dorsal es la recomendada, la elevación o no de la cabeza, es controversial. Se recomienda que la posición decúbito lateral derecho en las primeras horas post alimentación ayuda al vaciamiento gástrico y luego debiera cambiarse a decúbito lateral izquierdo para disminuir el reflujo.

\section{En adolescentes}

Las recomendaciones para ellos han sido extrapoladas de los estudios de adultos.

La pérdida de peso en los con sobrepeso mejora los síntomas e índices de reflujo.

La eliminación del tabaco y el alcohol tienen un efecto favorable en la reducción del reflujo.

No se ha confirmado que la ingesta de otros alimentos sea capaz de reducir la presión del EII, disminuyendo así los síntomas e índice de RGE. Sin embargo, la recomendación de expertos es disminuir cafeína, chocolate y condimentos.

Los adultos que comen más tarde en la noche, tienen más reflujo, que los que comen más temprano.

La posición más indicada en los adolescentes es dormir decúbito lateral izquierdo con cabecera levantada.

\section{Terapia farmacológica \\ Antiácidos:}

Deben ser usados por cortos períodos para aliviar el dolor y la pirosis.

Su uso prolongado aumenta niveles de aluminio, lo que causa osteopenia, anemia microcitica y neurotoxicidad.

\section{Cito protectores (sucralfato):}

Se adhiere a las lesiones pépticas, es útil en esofagitis con lesiones endoscópicas. Requiere para su acción un medio ácido. Sin embargo, como es un complejo con aluminio tiene mayor riesgo de reacciones adversas.

\section{Antagonistas de los receptores histamina-2}

Disminuyen la secreción gástrica inhibiendo los receptores en las células parietales gástricas. 
La ranitidina en dosis de 5 a $10 \mathrm{mg} / \mathrm{k} /$ día incrementa el $\mathrm{PH}$ gástrico. La vida media es de 2 horas. Inicia su efecto a los 30 minutos y dura su efecto 6 horas.

Se describe taquifilaxia posterior a las 6 semanas, por lo cual no tiene indicación como uso crónico. Tiene efectos adversos como cefalea, irritabilidad, somnolencia.

La eficacia de la ranitidina es mayor en las esofagitis moderadas que en las severas, la tasa de curación de la esofagitis erosiva es de 60 a $70 \%$.

\section{Inhibidores de la bomba de protones}

Inhiben la bomba atepasa $\mathrm{Na}-\mathrm{K}$, que tiene interacción en una vía de producción de $\mathrm{HCl}$ de la célula parietal gástrica, llamada bomba de protones, su eficacia está relacionada con su habilidad de mantener elevado el $\mathrm{pH}$ gástrico por un período prolongado y suprime la secreción ácida inducida por el alimento lo que no ocurre con los antagonistas de los receptores de histamina.

Tienen muy buena respuesta en el tratamiento de la enfermedad por reflujo y en la esofagitis erosiva. Su efecto no disminuye con el tiempo. Se pueden usar 1 vez al día antes del desayuno. Además tienen efecto en disminuir el volumen gástrico, mejorar su vaciamiento y disminuir el volumen refluido.

Deben ser ingeridos con protección entérica, su efecto puede durar hasta 4 días. No existe aprobación para su uso en los menores de un año. Entre 1-10 años requieren mayor dosis en proporción a su peso que en adultos y adolescentes.

Se describen efectos adversos que pueden ser idiosincráticos (cefalea, náuseas, constipación o diarrea), por interacción de droga-droga, por producción de hipergastrinemia (hiperplasia de las células parietales, pólipos gástricos); por hipoclorhidria (mayor frecuencia de neumonía adquirida, candidemia, gastroenteritis, enterocolitis en pre términos).

Otros efectos adversos vistos en adultos mayores son nefritis intersticial y deficiencia de vitamina B12.

\section{Procinéticos \\ Según las recientes revisiones no hay evi-}

dencia sustantiva que justifique el uso de los distintos procinéticos, sumados a los efectos colaterales que estos tienen. Se incluye domperidona, metoclopramida, baclofen, eritromicina y cisaprida.

\section{Cirugía \\ Fundoplicadura}

Reduce reflujo incrementando presión del LES, disminuyendo relajaciones transitorias y relajaciones asociadas a deglución, incrementa el largo del esófago intraabdominal, acentúa ángulo de Hiss.

La cirugía anti reflujo puede ser beneficiosa en aquellos niños con enfermedad por reflujo confirmada en que una terapia médica optima ha fracasado o que dependen de esta por largo tiempo; cuando no hay adherencia al tratamiento o en complicaciones que comprometan la vida; también está indicada en pacientes neurológicos, o en aquellos casos en que se realiza junto a gastrostomía.

Otros casos como asmáticos, aspiración pulmonar recurrente relacionada con el reflujo, se benefician también con la cirugía, pero debe estudiarse y confirmarse esta condición

Es importante explicar a la familia sobre las complicaciones de la cirugía y la posible recurrencia de los síntomas.

\section{Referencias}

1.- Vandenplas $Y$, Rudolphz CD, Di Lorenzo C, et al: Pediatric Gastro esophageal Reflux Clinical Practice Guidelines: Joint Recommendations of the North American Society for Pediatric Gastroenterology, Hepatology, and Nutrition (NASPGHAN) and the European Society for Pediatric Gastroenterology, Hepatology, and Nutrition (ESPGHAN) Journal of Pediatric Gastroenterology and Nutrition 2009; 49: 498-547.

2.- Garza JM, MD, Kaul A, MD: Gastroesophageal Reflux, Eosinophilic Esophagitis, and Foreign Body Pediatr Clin N Am 2010; 57: 1331-45

3.- Sherman PM, MD, Hassall E, MD, Fagundes-Neto $U$, MD, et al: Am J Gastroenterol 2009; 104: 1278-95.

4.- Dranove JE, MD: New technologies for the diagnosis of gastroesophageal reflux disease Pediatrics in Review 2008; 29: 317-320.

5.- Suwandhi E, MD, Ton MN, MD, Schwarz SM, MD: Gastro esophageal reflux in infancy and childhood Pediatric 
Annals 2006; 35: 259-66.

6.- Vandenplas Y, Salvatore S, Devreker T, et al: Gastroesophageal reflux disease: esophageal impedance versus pH monitoring Acta Pediatrica 2007; 96: 956-62.

7.- Di Pace MR, Caruso AM, Catalano P, Casuccio A, De Gracia E: Evaluation of Esofaphageal Motility Using Multichannel Intraluminal Impedance in Healthy Children and Children with Gastroesophageal Reflux. Jounal Pediatric Gastroenterology and Nutrition 2011; 52: 26-30.

8.- Mc Coul ED, MD MPH, Goldstein NA, MD, MPH,
Oliskor B, BA, et al: Prospective Study of Effect of Gastroesophageal Reflux Disease Treatment on Children With Otitis Media Arch Otolaryngol Head Neck Surg 2011; 137 (1): 35-41.

9.- Garza JM, MD, Kaul A, MD: Gastro esophageal Reflux, Eosinophilic Esophagitis, and Forein Body. Pediatric Clin N Am 2010; 57: 1331-45.

10.- Ji Hyuk Lee, Mi Jin Kim, Jong Seung Lee, Yon Ho Choe: The Effects of three alternative treatment strategies after 8 weeks of proton pump inhibitor therapy for GERD in children. Arch Dis Child 2011; 96: 9-13. 